\title{
What can be expected from the GRACE-FO Laser Ranging Interferometer for Earth Science applications?
}

Frank Flechtner (1), Karl-Hans Neumayer (1), Christoph Dahle (1), Henryk Dobslaw (1), Andreas Güntner (1), Jean-Claude Raimondo (2), and Elisa Fagiolini (1)

(1) Helmholtz Centre Potsdam, GFZ German Research Centre for Geosciences, Potsdam, Germany

(flechtne@gfz-potsdam.de), (2) SpaceTech GmbH, Immenstaad, Germany

The primary objective of the Gravity Recovery and Climate Experiment Follow-on (GRACE-FO) satellite mission is to continue the GRACE time series of global monthly gravity field models. For this, evolved versions of the GRACE microwave instrument (MWI), GPS-receiver, and accelerometer will be used. A secondary objective is to demonstrate the effectiveness of a laser ranging interferometer (LRI) in improving the low-low satellite-to-satellite tracking measurement performance.

In order to investigate the expected benefit of the LRI for Earth science applications, we performed a fullscale simulation in terms of spherical harmonics over the nominal mission lifetime of five years using a realistic orbit scenario and error assumptions for orbit, instrument and background model errors.

We will present results in the spectral and spatial domain showing moderate improvements when using LRI instead of MWI observations for global quality indicators. As these global indicators are not meaningful for Earth system applications which show a clear mass variation signal in regionally defined areas such as water mass changes in hydrological basins or melting of glaciers we have also additionally investigated how simulated seasonal, sub-seasonal, secular and instantaneous (Earthquake) signals are recovered when using GRACE-FO MWI or LRI data. Related results will be presented at the conference.

Analysis of the different individual error contributions to the overall monthly gravity model error has shown that dominant errors are still due to accelerometer noise and imperfect modeling of tidal and non-tidal mass variations. Consequently, these errors have to be further reduced when using LRI observations on Next Generation Gravity Missions. 